\title{
ENERGETICS AND SWITCHING OF QUASI-UNIFORM STATES IN SMALL FERROMAGNETIC PARTICLES
}

\author{
François Alouges ${ }^{1}$, Sergio Conti ${ }^{2}$, Antonio DeSimone ${ }^{3}$ And Yvo Pokern ${ }^{4}$
}

\begin{abstract}
We present a numerical algorithm to solve the micromagnetic equations based on tangential-plane minimization for the magnetization update and a homothethic-layer decomposition of outer space for the computation of the demagnetization field. As a first application, detailed results on the flower-vortex transition in the cube of Micromagnetic Standard Problem number 3 are obtained, which confirm, with a different method, those already present in the literature, and validate our method and code. We then turn to switching of small cubic or almost-cubic particles, in the single-domain limit. Our data show systematic deviations from the Stoner-Wohlfarth model due to the non-ellipsoidal shape of the particle, and in particular a non-monotone dependence on the particle size.
\end{abstract}

Mathematics Subject Classification. 65L60, 78M10, 82D40.

Received: October 28, 2003.

\section{INTRODUCTION}

Small magnetic particles have been the subject of intense theoretical and experimental research for many decades, and interest has recently been revived by the increasing relevance for magnetic recording systems [6,16]. At the same time, improvements in experimental techniques have led to the possibility of observing individual particles $[9,20]$, while increased computational capabilities have permitted numerical calculations in situations which are not amenable to complete analysis by traditional theoretical methods.

We present here the first application to real micromagnetic problems of a new finite-element scheme, which has been proposed by Alouges in [4]. Computing micromagnetic equilibria amounts to solving a nonlocal, constrained minimization problem. Our approach is based on computing the demagnetization field on a homothetical decomposition of a finite portion of the outer space, whose mesh is generated by scaling the surface mesh of the body (or of a convex set that contains the magnetic body). The nonlinear update of the magnetization is then obtained by minimization on the manifold tangent to the constraint. This is a linear problem, at least for quadratic anisotropy. This update method has been already used in the context of liquid crystals in [3].

\footnotetext{
Keywords and phrases. Micromagnetics, finite elements.

1 Laboratoire de Mathématique, Université d'Orsay, 91405 Orsay Cedex, France, and

Centre de Mathématiques et de Leurs Applications, École Normale Supérieure de Cachan, 61 avenue du Président Wilson, 94235 Cachan Cedex, France. e-mail: francois.alouges@math.u-psud.fr

2 Max Planck Institute for Mathematics in the Sciences, Inselstr. 22, 04103 Leipzig, Germany. e-mail: Sergio.Conti@mis.mpg.de

3 SISSA, International School for Advanced Studies, Via Beirut 2-4, 34014 Trieste, Italy. e-mail: desimone@sissa.it

4 Department of Mathematics, University of Warwick, Coventry CV4 7AL, UK.
} 
Quasi-uniform states characterize the magnetic behavior of sufficiently small particles. A precise determination of the critical size at which the assumption of a quasi-uniform magnetization breaks down is, however, a nontrivial task. Indeed, starting from the pioneering work of Shabes and Bertram [23], a large numerical literature has developed on the subject $([15,16,21]$ and references therein), and a specific set of parameters has been chosen as Standard Problem No. 3 [18] for the validation of numerical micromagnetic algorithms. We discuss in Section 4 our results for the transition from the quasi-uniform flower state to the vortex state in this standard cube.

Switching in small particles analogously has a critical dependence on particle size. Most studies on small particles are based, in one way or another, on the Stoner-Wohlfarth (SW) model [24], which assumes uniform magnetization inside the particle $[1,16]$. One of the peculiarities of the SW model is that geometrical effects (i.e. the shape of the particle) enter only through a quadratic form, or equivalently that all small particles behave as ellipsoids. Using a Gamma-convergence argument, it has been shown in [12] that micromagnetic equilibria converge to solutions of the SW model when the size of the particle tends to zero. A question we wish to address with our computational method is that of deviations from SW behavior for particles which are small, but of finite size.

Ellipsoids have the property that a uniform magnetization is an exact equilibrium of the micromagnetic equations. A large theoretical literature exists on rigorous results for the linear stability of this uniform state in prolate or oblate ellipsoids, and on the different instability channels, see e.g. [1,2,8,14,17]. Switching is often associated with loss of linear stability. Even for ellipsoids, attention to the predictions of the SW model in cases without rotational symmetry is much more recent $[7,25]$, and a complete linear stability analysis is still missing. The question of the validity of the ellipsoidal approximation for non-ellipsoidal particles has attracted some attention [19,23], but this issue is far from being fully understood. In Section 5 we present numerical results for particles of cubic or almost-cubic shape, within the single-domain regime, which illustrate the leading corrections to the SW model with increasing particle size. For small sizes our critical fields reproduce the SW results. Larger particles have shape-dependent critical fields with a non-monotone dependence on size and with a complex angular dependence, which cannot be reproduced assimilating the particle to an ellipsoid.

\section{Model, Algorithm AND CONVERGEnCE}

We work in a quasistatic setting, and minimize the micromagnetic energy [16], which after scaling takes the form

$$
E[m]=\int_{\Omega} A|\nabla m|^{2}+K_{u} m_{y}^{2}-h_{\mathrm{ext}} \cdot m-\frac{1}{2} h_{\mathrm{d}} \cdot m
$$

Here $|m|=1$ in $\Omega$ and 0 outside, $h_{\text {ext }}$ is the (uniform) applied field, $K_{u}$ is the uniaxial anisotropy constant, $A$ is the exchange constant and $h_{\mathrm{d}}=H(m)$ is the stray-field, which is defined as the $L^{2}$-orthogonal projection of $-m$ on gradient vector fields. Precisely, the linear operator $H$ satisfies

$$
\begin{aligned}
& \quad \forall v, w \in L^{2}\left(\Omega, \mathbb{R}^{3}\right) \int_{\mathbb{R}^{3}} H(v) \cdot H(w)=-\int_{\Omega} H(v) \cdot w \\
& \text { and } \quad \forall v \in L^{2}\left(\Omega, \mathbb{R}^{3}\right) \int_{\mathbb{R}^{3}}|H(v)|^{2} \leq \int_{\Omega}|v|^{2}
\end{aligned}
$$

Equivalently, one can first solve $\Delta \psi=\nabla \cdot m$ in $\mathbb{R}^{3}$ and then compute $h_{\mathrm{d}}=-\nabla \psi$.

The corresponding Euler-Lagrange equation can be obtained taking a smooth variation $\phi \in \mathcal{C}_{0}^{\infty}(\Omega)$ and writing

$$
\left.\frac{\mathrm{d}}{\mathrm{d} \lambda} E\left[\frac{m+\lambda \phi}{|m+\lambda \phi|}\right]\right|_{\lambda=0}=0
$$


This gives

$$
-A \Delta m+K_{u}\left(\begin{array}{c}
0 \\
m_{y} \\
0
\end{array}\right)-\frac{h_{\mathrm{d}}+h_{\mathrm{ext}}}{2}=\left(A|\nabla m|^{2}+K_{u} m_{y}^{2}-\frac{h_{\mathrm{d}}+h_{\mathrm{ext}}}{2} \cdot m\right) m
$$

which is understood in the sense of distributions. A magnetization distribution that satisfies (4) will be called hereafter a critical point of the energy. As for harmonic maps into spheres (see for instance [11]), critical points with finite energy equivalently satisfy the following equation in the sense of distributions

$$
-A \operatorname{div}(\nabla m \wedge m)+K_{u}\left(\begin{array}{c}
0 \\
m_{y} \\
0
\end{array}\right) \wedge m-\frac{h_{\mathrm{d}}+h_{\mathrm{ext}}}{2} \wedge m=0
$$

Before dealing with discretization details, we explain the algorithm that is used. It is essentially a one-step method in the sense that we build a sequence $\left(m^{(n)}\right)$ of magnetizations satisfying the constraint $\left|m^{(n)}(x)\right|=1$ a.e. and such that for each $n$

$$
E\left[m^{(n+1)}\right] \leq E\left[m^{(n)}\right]
$$

The construction of $m^{(n+1)}$ from $m^{(n)}$ is done in two steps

- Compute a descent direction $w^{(n)}$,

- Update the magnetization by setting

$$
m^{(n+1)}(x)=\frac{m^{(n)}(x)+\lambda_{n} w^{(n)}(x)}{\left|m^{(n)}(x)+\lambda_{n} w^{(n)}(x)\right|},
$$

with a suitable value of $\lambda_{n}$.

The descent direction $w^{(n)}$ used in the first step of the algorithm is computed as the $H^{1}$-gradient of the energy. Precisely, if $m$ is the magnetization, following [3] we call

$$
T_{m}=\left\{w \in H^{1}\left(\Omega, \mathbb{R}^{3}\right), \text { such that } w(x) \cdot m(x)=0 \text { a.e. }\right\},
$$

the subspace of $H^{1}\left(\Omega, \mathbb{R}^{3}\right)$ which contains variations tangent to the constraint around $m$. It is easily seen that $T_{m}$ is closed in $H^{1}\left(\Omega, \mathbb{R}^{3}\right)$ with respect to weak and strong convergence. We then seek $w$ as the solution to the problem

$$
\left\{\begin{array}{l}
w \in T_{m}, \\
\frac{\mathrm{d}}{\mathrm{d} \lambda} E[m+\lambda \phi]_{\left.\right|_{\lambda=0}}=-2 A\left(\int_{\Omega} \nabla w \cdot \nabla \phi+\int_{\Omega} w \cdot \phi\right), \forall \phi \in T_{m} .
\end{array}\right.
$$

(The minus sign on the right hand side directly gives $w$ in the opposite direction to the gradient of the energy.) Existence and uniqueness of $w$ is a classical matter, and we simply rewrite the equation as

$$
\left\{\begin{aligned}
w \in T_{m} & \\
\int_{\Omega}[2 A \nabla(m+w) \cdot \nabla \phi & +2 A(m+w) \cdot \phi+2 K_{u} m_{y} \phi_{y} \\
& \left.-h_{\mathrm{ext}} \cdot \phi-H(m) \cdot \phi\right]=0, \quad \forall \phi \in T_{m}
\end{aligned}\right.
$$

As observed in [3] solving this equation acts like a preconditioner on the original system and the convergence is therefore much better when compared with a classical gradient method.

Remark 1. Taking $\phi=w$ in the expression above yields an estimate on the $H^{1}$ norm of $w$. Indeed, we easily get

$$
2 A\|w\|_{H^{1}(\Omega)}^{2} \leq\left(\left(2 A+2 K_{u}+1\right)\|m\|_{H^{1}(\Omega)}+\left|h_{\mathrm{ext}}\right||\Omega|^{\frac{1}{2}}\right)\|w\|_{H^{1}(\Omega)},
$$


from which we deduce the following bound on $\|w\|_{H^{1}(\Omega)}$ which will be useful in the next part

$$
\|w\|_{H^{1}(\Omega)} \leq \alpha\|m\|_{H^{1}(\Omega)}+\beta,
$$

where $\alpha$ and $\beta$ are explicit constants. From (6) we then obtain a uniform bound for the sequence $\left(w^{(n)}\right)$,

$$
\left\|w^{(n)}\right\|_{H^{1}(\Omega)} \leq C .
$$

Returning to the definition of the sequence, we have the following theorem.

Theorem 2. There exists $\lambda_{0}>0$ which depends only on $\Omega, A, K_{u}$, and $h_{\mathrm{ext}}$ such that the sequence $\left(\mathrm{m}^{(n)}\right)$ generated by the algorithm for any $\lambda \leq \lambda_{0}$ converges (up to the extraction of a subsequence) weakly in $H^{1}(\Omega$ ) to a critical point of the energy.

The proof follows the strategy used in [3] which mainly consists in passing to the limit in equation (7).

We start with the estimation of $E\left[m_{\lambda}\right]-E[m]$, where $m_{\lambda}=\frac{m+\lambda w}{|m+\lambda w|}$, and use the following lemma.

Lemma 3. Let $m \in H^{1}\left(\Omega, S^{2}\right)$. If $w \in H^{1}\left(\Omega, \mathbb{R}^{3}\right)$ is a vector field such that $w(x) \cdot m(x)=0$ a.e., then

$$
\int_{\Omega}\left|\nabla m_{\lambda}\right|^{2} \leq \int_{\Omega}|\nabla(m+\lambda w)|^{2}
$$

Proof. The statement follows pointwise from the following fact: if $e \in S^{2}$, and $\rho \geq 1$, then $|\nabla(\rho e)|^{2}=\rho^{2}|\nabla e|^{2}+$ $|\nabla \rho|^{2} \geq|\nabla e|^{2}$.

Proof of Theorem 2. We estimate the energy decay

$$
\begin{aligned}
E\left[m_{\lambda}\right]-E[m]= & A \int_{\Omega}\left|\nabla m_{\lambda}\right|^{2}+K_{u} \int_{\Omega}\left(m_{\lambda, y}\right)^{2}-\int_{\Omega} h_{\mathrm{ext}} \cdot m_{\lambda} \\
& +\frac{1}{2} \int_{\mathbb{R}^{3}}\left|H\left(m_{\lambda}\right)\right|^{2}-A \int_{\Omega}|\nabla m|^{2}-K_{u} \int_{\Omega} m_{y}^{2} \\
& +\int_{\Omega} h_{\mathrm{ext}} \cdot m-\frac{1}{2} \int_{\mathbb{R}^{3}}|H(m)|^{2} \\
\leq & A \int_{\Omega}\left(|\nabla(m+\lambda w)|^{2}-|\nabla m|^{2}\right)+K_{u} \int_{\Omega}\left(m_{y}+\lambda w_{y}\right)^{2}-m_{y}^{2} \\
& -\int_{\Omega} h_{\mathrm{ext}} \cdot\left(m_{\lambda}-m\right)+\frac{1}{2} \int_{\mathbb{R}^{3}}\left|H\left(m_{\lambda}\right)\right|^{2}-|H(m)|^{2},
\end{aligned}
$$

from the estimate given in Lemma 3. Using again (7) with $\phi=w$, we get

$$
A \int_{\Omega} \nabla m \cdot \nabla w+K_{u} \int_{\Omega} m_{y} w_{y}=-A \int_{\Omega}\left(|\nabla w|^{2}+|w|^{2}\right)+\int_{\Omega} \frac{h_{\mathrm{ext}}+H(m)}{2} \cdot w,
$$

from which the estimate becomes

$$
\begin{aligned}
E\left[m_{\lambda}\right]-E[m] \leq & \left(-2 A \lambda+A \lambda^{2}\right) \int_{\Omega}|\nabla w|^{2}-2 A \lambda \int_{\Omega}|w|^{2}+K_{u} \lambda^{2} \int_{\Omega} w_{y}^{2} \\
& -\int_{\Omega} h_{\mathrm{ext}} \cdot m_{\lambda}(1-|m+\lambda w|)+\frac{1}{2} \int_{\mathbb{R}^{3}}\left|H\left(m_{\lambda}\right)\right|^{2} \\
& -\frac{1}{2} \int_{\mathbb{R}^{3}}|H(m)|^{2}+\lambda \int_{\Omega} H(m) \cdot w .
\end{aligned}
$$


Now we estimate the terms on the right hand side. First, due to $w(x) \cdot m(x)=0$ a.e., we have

$$
|1-| m(x)+\lambda w(x)|| \leq \frac{\lambda^{2}}{2}|w(x)|^{2}
$$

from which we deduce

$$
\left|\int_{\Omega} h_{\mathrm{ext}} \cdot m_{\lambda}(1-|m+\lambda w|)\right| \leq \frac{\lambda^{2}}{2}\left|h_{\mathrm{ext}}\right| \int_{\Omega}|w|^{2} .
$$

Now we consider the term involving the stray-field energy, and decompose it into two terms

$$
\begin{aligned}
\frac{1}{2} \int_{\mathbb{R}^{3}}\left|H\left(m_{\lambda}\right)\right|^{2}-|H(m)|^{2}+\lambda \int_{\Omega} H(m) \cdot w= & \frac{1}{2} \int_{\mathbb{R}^{3}}\left|H\left(m_{\lambda}\right)\right|^{2}-|H(m+\lambda w)|^{2} \\
& +\frac{1}{2} \int_{\mathbb{R}^{3}}|H(m+\lambda w)|^{2}-|H(m)|^{2}+\lambda \int_{\Omega} H(m) \cdot w \\
= & \frac{1}{2} \int_{\mathbb{R}^{3}}\left|H\left(m_{\lambda}\right)\right|^{2}-|H(m+\lambda w)|^{2} \\
& +\frac{\lambda^{2}}{2} \int_{\mathbb{R}^{3}}|H(w)|^{2} .
\end{aligned}
$$

From (3) we get

$$
\frac{\lambda^{2}}{2} \int_{\mathbb{R}^{3}}|H(w)|^{2} \leq \frac{\lambda^{2}}{2} \int_{\Omega}|w|^{2}
$$

The remaining term can be written as

$$
\begin{aligned}
\int_{\mathbb{R}^{3}}\left|H\left(m_{\lambda}\right)\right|^{2}-|H(m+\lambda w)|^{2} & =\int_{\mathbb{R}^{3}}\left(H\left(m_{\lambda}\right)+H(m+\lambda w)\right) \cdot\left(H\left(m_{\lambda}\right)-H(m+\lambda w)\right) \\
& =-\int_{\Omega}\left(H\left(m_{\lambda}\right)+H(m+\lambda w)\right) \cdot\left(m_{\lambda}-(m+\lambda w)\right),
\end{aligned}
$$

where we used (2). Using the Cauchy-Schwarz inequality and (3),

$$
\begin{aligned}
\left.\left|\int_{\mathbb{R}^{3}}\right| H\left(m_{\lambda}\right)\right|^{2}-|H(m+\lambda w)|^{2} \mid & \leq\left\|H\left(m_{\lambda}\right)+H(m+\lambda w)\right\|_{L^{2}\left(\mathbb{R}^{3}\right)}\left\|m_{\lambda}-(m+\lambda w)\right\|_{L^{2}(\Omega)} \\
& \leq\left(|\Omega|^{\frac{1}{2}}+\|m+\lambda w\|_{L^{2}(\Omega)}\right) \frac{\lambda^{2}}{2}\|w\|_{L^{4}(\Omega)}^{2} \\
& \leq C_{1}^{2}\left(|\Omega|^{\frac{1}{2}}+\frac{1}{2} \lambda\|w\|_{H^{1}(\Omega)}\right) \lambda^{2}\|w\|_{H^{1}(\Omega)}^{2}
\end{aligned}
$$

where $C_{1}$ is the continuity constant of the embedding $H^{1}(\Omega) \rightarrow L^{4}(\Omega)$. 
Putting everything together leads to

$$
\begin{aligned}
E\left[m_{\lambda}\right]-E[m] \leq & \left.-2 A \lambda+A \lambda^{2}\right) \int_{\Omega}|\nabla w|^{2}-2 A \lambda \int_{\Omega}|w|^{2}+K_{u} \lambda^{2} \int_{\Omega} w_{y}^{2} \\
& +\frac{\lambda^{2}}{2}\left(\left|h_{\mathrm{ext}}\right|+1\right) \int_{\Omega}|w|^{2}+C_{1}^{2}\left(|\Omega|^{\frac{1}{2}}+\frac{1}{2} \lambda\|w\|_{H^{1}(\Omega)}\right) \lambda^{2}\|w\|_{H^{1}(\Omega)}^{2} \\
\leq & \left(-2 A \lambda+\left(A+K_{u}+\frac{\left|h_{\mathrm{ext}}\right|+1}{2}\right) \lambda^{2}\right)\|w\|_{H^{1}(\Omega)}^{2} \\
& \quad+C_{1}^{2}\left(|\Omega|^{\frac{1}{2}}+\frac{1}{2} \lambda\|w\|_{H^{1}(\Omega)}\right) \lambda^{2}\|w\|_{H^{1}(\Omega)}^{2} \\
\leq & \left(-2 A \lambda+\left(A+K_{u}+\frac{\left|h_{\mathrm{ext}}\right|+1}{2}+C_{1}^{2}|\Omega|^{\frac{1}{2}}\right) \lambda^{2}+C_{2} \lambda^{3}\right)\|w\|_{H^{1}(\Omega)}^{2}
\end{aligned}
$$

where $C_{2}$ depends on the constants $|\Omega|, A, K_{u}$ and $\|m\|_{H^{1}(\Omega)}$ through the bound (8). At the same time, $\|m\|_{H^{1}(\Omega)}$ is controlled by the energy $E[m]$.

From the signs of the coefficients, it is clear that the polynomial function of degree three in $\lambda$ that appears on the right hand side of the inequality is negative for $\lambda \in\left(0, \lambda_{0}\right)$, for some $\lambda_{0}>0$ which depends only on the parameters of the problem and $E[m]$. That for small $\lambda$ the energy is reduced was clear a priori, since the first step of the algorithm is essentially a gradient method whereas the renormalization stage only modifies the magnetization by a second order term in $\lambda$. We have, additionally, obtained a quantitative relation between the reduction of the energy and the norm of $w$.

If at each iteration step we choose $\lambda<\lambda_{0}$, by (6) the energy is nonincreasing. In turn, this implies that $C_{2}$ above can be taken constant along the sequence, estimating $E\left[m^{(n)}\right] \leq E\left[m^{(0)}\right]$. Then, the polynomial is the same along the sequence. Choose now a single $\lambda<\lambda_{0}$, and let $C^{\lambda}$ be the corresponding value of the polynomial. This gives a quantitative version of (6), namely,

$$
E\left[m_{\lambda}\right]-E[m] \leq-C^{\lambda}\|w\|_{H^{1}(\Omega)}^{2}
$$

(recall that the sequence is defined by $m^{(n+1)}=m_{\lambda}$, if $m=m^{(n)}$ ). At the same time, since the energy is bounded from below, the series

$$
\sum_{n>0}\left\|w^{(n)}\right\|_{H^{1}(\Omega)}^{2}
$$

which is controlled by the total energy decay must converge.

We finish the reasoning by passing to the limit in (7), to show that the limit is indeed a stationary point of the energy. Substituting $m^{(n)}$ and $w^{(n)}$ we get

$$
\begin{aligned}
A \int_{\Omega} \nabla\left(m^{(n)}+w^{(n)}\right) \cdot \nabla \psi+A \int_{\Omega}\left(m^{(n)}+w^{(n)}\right) \cdot \psi+K_{u} & \int_{\Omega} m_{y}^{(n)} \psi_{y} \\
& =\int_{\Omega} h_{\mathrm{ext}} \cdot \psi+\int_{\Omega} H\left(m^{(n)}\right) \cdot \psi \quad \forall \psi \in T_{m^{(n)}} .
\end{aligned}
$$

We first notice that the sequence $\left(m^{(n)}\right)$ is uniformly bounded in $H^{1}(\Omega)$ and we may therefore extract a subsequence from it (still denoted by $\left(m^{(n)}\right)$ ) such that

$$
\begin{gathered}
m^{(n)} \rightarrow m^{(\infty)}, \text { weakly in } H^{1}(\Omega) \\
m^{(n)} \rightarrow m^{(\infty)}, \text { strongly in } L^{2}(\Omega) .
\end{gathered}
$$


Since $H$ is a continuous operator on $L^{2}(\Omega)$ we deduce

$$
H\left(m^{(n)}\right) \rightarrow H\left(m^{(\infty)}\right) \text {, strongly in } L^{2}(\Omega),
$$

and from the convergence of the series $\sum_{n}\left\|w^{(n)}\right\|_{H^{1}(\Omega)}^{2}$,

$$
w^{(n)} \rightarrow 0, \text { strongly in } H^{1}(\Omega) .
$$

Now, take $\psi=m^{(n)} \wedge \phi$ in (12). We obtain

$$
\begin{aligned}
\int_{\Omega} A\left[\nabla\left(m^{(n)}+w^{(n)}\right) \cdot\left(m^{(n)} \wedge \nabla \phi\right)\right. & \left.+\nabla w^{(n)} \cdot\left(\nabla m^{(n)} \wedge \phi\right)+\left(m^{(n)}+w^{(n)}\right) \cdot\left(m^{(n)} \wedge \phi\right)\right] \\
& +K_{u} \int_{\Omega}\left(m_{y}^{(n)}\right)\left(m^{(n)} \wedge \phi\right)_{y}=\int_{\Omega} h_{\mathrm{ext}} \cdot\left(m^{(n)} \wedge \phi\right)-\int_{\Omega} H\left(m^{(n)}\right) \cdot\left(m^{(n)} \wedge \phi\right)
\end{aligned}
$$

for all $\phi \in C_{0}^{\infty}(\Omega)$. All the terms pass to the limit, and we deduce

$$
\begin{aligned}
& A \int_{\Omega} \nabla m^{(\infty)} \cdot\left(m^{(\infty)} \wedge \nabla \phi\right)+K_{u} \int_{\Omega} m_{y}^{(\infty)}\left(m^{(\infty)} \wedge \phi\right)_{y}=\int_{\Omega} h_{\mathrm{ext}} \cdot\left(m^{(\infty)} \wedge \phi\right) \\
&-\int_{\Omega} H\left(m^{(\infty)}\right) \cdot\left(m^{(\infty)} \wedge \phi\right) \quad \forall \phi \in C_{0}^{\infty}(\Omega),
\end{aligned}
$$

which means from (5) that $m^{(\infty)}$ is a critical point of the energy.

Remark 4. Several alternatives may be used to compute the descent direction $w$. Instead of taking the $H^{1}$-inner product, one may for instance add the anisotropy term. It doesn't change the convergence result but may help to decrease the energy more quickly. In the numerical applications described in the next section, we use this alternative.

\section{NumericAl METHOD}

The numerical minimization of $E[m]$ is an optimization problem with a non-convex constraint, which involves the computation of a quantity (the stray-field $h_{\mathrm{d}}$ ) in the whole space $\mathbb{R}^{3}$. Different methods have been proposed to treat each of these issues, depending on the details of the problem at hand. For instance, the series of papers by Fredkin and Koehler $([10,13]$ and references therein) systematically studied several discretization techniques in 2 dimensional and 3 dimensional cases. Other numerical techniques mainly based on the fast Fourier transform technique on regular grids have been employed (see e.g. $[5,21,22]$ ). Several benchmarks (one of which is studied in the next section) may be found on the NIST webpage [18].

The algorithm we have used is the one described in the preceding section. It is a preconditioned projected gradient method which iteratively builds a sequence $\left(m^{(n)}\right)$ of magnetizations in two steps:

- $m^{(n)}$ being known, compute a descent direction $w^{(n)}$;

- Update the magnetization by setting

$$
m^{(n+1)}(x)=\frac{m^{(n)}(x)+\lambda_{n} w^{(n)}(x)}{\left|m^{(n)}(x)+\lambda_{n} w^{(n)}(x)\right|},
$$

with a suitable value of $\lambda_{n}$. 
The computation of the descent direction $w^{(n)}$ is done as the solution to the minimization problem on $T_{m}(n)$ (compare with (7))

$$
\min _{w \in T_{m}(n)} \int_{\Omega} A\left|\nabla\left(m^{(n)}+w\right)^{2}\right|+K_{u}\left(m_{y}^{(n)}+w_{y}\right)^{2}-h_{\mathrm{ext}} \cdot\left(m^{(n)}+w\right)-h_{\mathrm{d}}^{(n)} \cdot\left(m^{(n)}+w\right) .
$$

Here, we have called $h_{\mathrm{d}}^{(n)}=H\left(m^{(n)}\right)$ the stray field induced by $m^{(n)}$. This problem is the minimization of a quadratic functional on a linear space. It therefore possesses a unique solution. After discretization with e.g. finite elements, it can be solved using a conjugate gradient method.

The new value for the magnetization is then obtained during the renormalization stage of the algorithm. A trial value $\lambda_{n}=1$ is chosen and the real energy $E\left[m^{(n+1)}\right]$ is computed. If the real energy has decreased, i.e. $E\left[m^{(n+1)}\right]<E\left[m^{(n)}\right]$, we accept the update and proceed with the next iteration. Otherwise we reduce $\lambda_{n}$ (by a factor of 2 ), and determine a new $m^{(n+1)}$ (without having to recompute $w^{(n)}$ ). This is roughly equivalent to a $\lambda$-strategy in a Newton algorithm. Typically $\lambda_{n}$ oscillates between $1 / 2$ and 1 , only in a few cases it has gone down to $\frac{1}{4}$. The algorithm stops when the update $w^{(n)}$ falls below a certain threshold (sum of the squares of the entries less than $10^{-8}$ or $10^{-10}$ ).

For the discretization, we used the so-called $P^{1}-$ finite element technique. The domain $\Omega$ is meshed into tetrahedra and the magnetization is then represented using affine functions for each component, with the unitlength constraint enforced at the nodes.

We now turn to the computation of the stray field $h_{\mathrm{d}}$. To solve $\Delta \phi=\nabla \cdot m$ in $\mathbb{R}^{3}$ we use a technique recently proposed by Alouges [4] which appeared to be a viable alternative to the classical boundary elements methods. The demagnetizing potential is obtained by solving Poisson's equation on a much larger domain (ideally, $\mathbb{R}^{3}$ ). This is done using the homothetic-layers construction of Ying [4,26]. More precisely, we first build a mesh (with tetrahedra) for the region contained between $\Omega$ and a larger convex domain $\tilde{\Omega}$, whose linear dimensions are approximately twice those of $\Omega$. This allows to refine selectively both meshes around the boundary of $\Omega$, and hence to resolve e.g. the singularities at the cube corners, without having to resort to high resolution everywhere. At the same time we can construct the outer grid starting from the convex domain $\tilde{\Omega}$, without requiring the magnetic body $\Omega$ to be convex itself. Then, following [4], the tetrahedral mesh on the outer boundary of $\tilde{\Omega}$ is scaled up homothetically. From each vertex $x_{i} \in \tilde{\Omega}$ we get a sequence $x_{i}^{(j)}=\xi^{j} x_{i}, 1 \leq j \leq N$. This gives a meshing of the volume contained in $\xi^{N} \tilde{\Omega} \backslash \tilde{\Omega}$ with modified prisms. The outer mesh is obtained by carving up each of these prisms into three tetrahedra. As remarked by $[4,26]$, the homothetic structure of the mesh reflects into the matrix elements for the linear problem. The matrices need to be computed (and stored) explicitly only for the first layer, the outer layers are afterwards obtained by scaling. Following [4] we instead scale the unknowns corresponding to the outer nodes, leading to an automatic pre-conditioning of the Poisson problem, which significantly improves the performance of our Conjugate-Gradient linear solver. As boundary conditions we impose $\phi=0$ on the boundary of $\tilde{\Omega}$. On the boundary of $\Omega$ the magnetization is discontinuous, leading to singular contributions to the right-hand side of $\Delta \phi=\operatorname{div} m$, which have been treated as surface integrals.

In practice, we have used grids with average edge length $h$ ranging from 0.03 to 0.15 for the unit cube, which corresponds to 400 to 8000 nodes for the discretization of the magnetization. The typical edge length $h_{o}$ for the mesh of $\tilde{\Omega}$ was 2 to 3 times larger. The outer mesh was then generated using $\xi=1+2 h_{o}$, which corresponds to fixing the aspect ratio of the homothetic tetrahedra. The number of layers $N$ was chosen so that the total volume had linear dimensions around 100 times larger than $\Omega$. This ensures that the finite-volume correction is negligible. The above algorithm has been implemented in C++ on a parallel Sun machine. A typical minimization requires a few dozen nonlinear iterations, and lasts a few hours. 


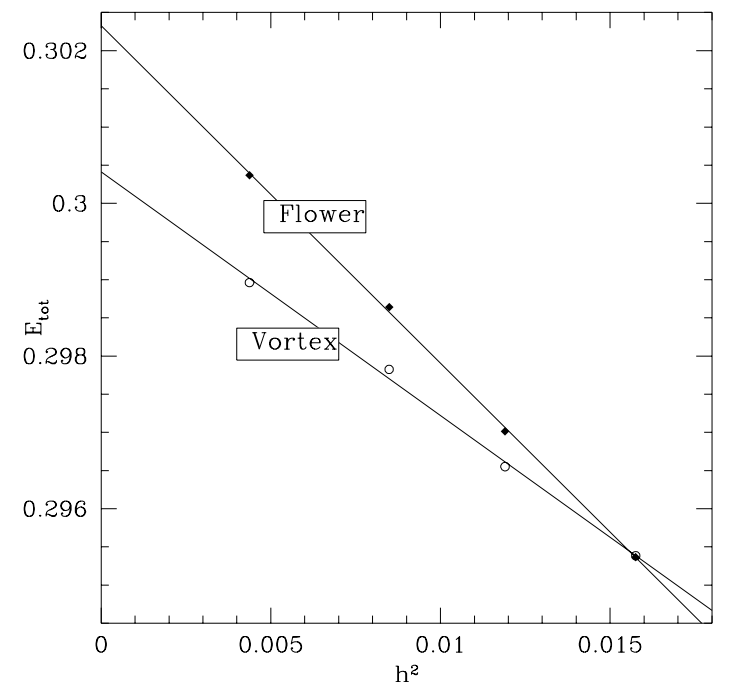

FiguRE 1. Grid-size extrapolation for $\lambda=8.528$. The dots mark the computed points, the full lines the linear fit.

\section{Phase diagram of the Standard Cube}

As a first application of our method, we consider the Micromagnetic Standard Problem 3 [15, 18, 21]. This consists in estimating the size of a cube at which the ground state changes from a quasi-uniform (flower) to a vortex state, in the presence of uniaxial anisotropy with $K_{u}=0.1$. The problem had been proposed by A. Hubert, and a first solution has been published by Rave, Fabian and Hubert (RFH) [21]. Hertel and Kronmüller (HK) [15] in a successive solution, with a different method, discovered the presence of an additional "twisted flower" state, which is a modification of the symmetric flower breaking inversion symmetry, with lower energy than the flower in the region of interest. We shall study both the flower-vortex and the twisted flower-vortex transitions.

To assess the different regions of stability we determined the total energy of each state for various values of $\lambda$ (defined as the cube edge divided by the exchange length $(A / 2)^{1 / 2}$ ). Due to the observed energy differences being rather small, we had to perform mesh-size extrapolation to obtain reliable results. This has been done using a least-squares fit with $E(h)=E(0)+c h^{2}$, where $h$ is the average edge length of the considered mesh. A typical extrapolation plot is displayed in Figure 1. Note that in refining $h$ both the inner mesh (used to resolve the magnetization pattern) and the outer mesh (used to resolve the demagnetization field) are refined. The number of layers is determined for every mesh so that the total volume is kept a cube of size around 100 times the unit cube of interest. The restriction to a finite volume does not significantly affect the energy, since the dipolar energy decays as $1 / L^{3}$. We checked with a uniformly magnetized cube that the chosen number of layers does not affect the total energy by more than $10^{-5}$. Hence this effect is neglected in the following. The largest source of error we expect is the uncertainty in the mesh-size extrapolation. To estimate this error, for each set of data we computed the standard deviation of the computed points from the fitted straight line. The result was always around $10^{-4}$. As a check, we tried to remove in turn one of the data points from each linear fit, and to compute the standard deviation of the so-obtained set of extrapolated results. The resulting error estimate was again around $10^{-4}$. Therefore, we take this value as the error on the extrapolated values.

The phase diagram we obtain is given in Figure 2, where the energies of the different states are compared. The intersection is determined by fitting a straight line between the three computed points for each phase, and the error is given by standard error propagation. We observe a transition from flower to vortex for $\lambda_{F-V}=$ $8.475 \pm 0.004$, whereas RFH had $\lambda_{F-V}=8.47$ and HK $\lambda_{F-V}=8.52$. Results analogously close to those of RFH 


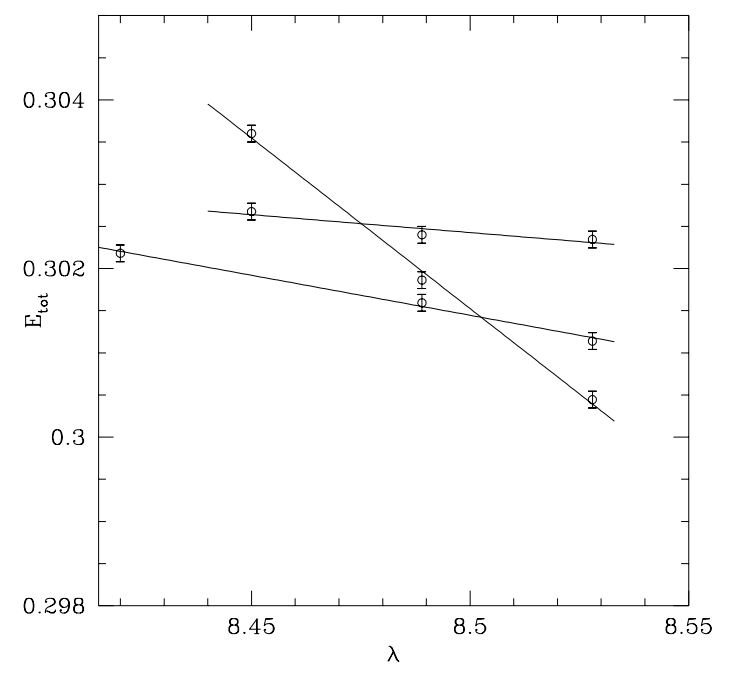

Figure 2. Energy as a function of $\lambda$ for the different states. The points are the grid-size extrapolated results. The curves are a linear interpolation used to determine the intersection.

TABLE 1. Partial energies per unit volume (demagnetization, exchange, anisotropy, and total) and average magnetizations for the (symmetric) flower state. The energy results are extrapolated to $h=0$ as discussed in the text. No mesh-size extrapolation was needed for the magnetization results. The last figure is likely to be affected by numerical errors.

\begin{tabular}{c|lllllll}
\hline$\lambda$ & $\varepsilon_{\mathrm{d}}$ & $\varepsilon_{\mathrm{x}}$ & $\varepsilon_{\mathrm{k}}$ & $\varepsilon_{\text {tot }}$ & $\left\langle m_{x}\right\rangle$ & $\left\langle m_{y}\right\rangle$ & $\left\langle m_{z}\right\rangle$ \\
\hline 8.528 & 0.2789 & 0.01776 & 0.00569 & 0.3023 & 0.000 & 0.970 & 0.000 \\
8.489 & 0.2791 & 0.01772 & 0.00564 & 0.3024 & 0.000 & 0.971 & 0.000 \\
8.450 & 0.2794 & 0.01768 & 0.00558 & 0.3027 & 0.000 & 0.971 & 0.000 \\
\hline
\end{tabular}

TABle 2. Vortex state. Same data as Table 1.

\begin{tabular}{c|lllllll}
\hline$\lambda$ & $\varepsilon_{\mathrm{d}}$ & $\varepsilon_{\mathrm{x}}$ & $\varepsilon_{\mathrm{k}}$ & $\varepsilon_{\text {tot }}$ & $\left\langle m_{x}\right\rangle$ & $\left\langle m_{y}\right\rangle$ & $\left\langle m_{z}\right\rangle$ \\
\hline 8.528 & 0.0771 & 0.1714 & 0.0520 & 0.3004 & 0.000 & 0.000 & 0.345 \\
8.489 & 0.0778 & 0.1720 & 0.0521 & 0.3019 & 0.000 & 0.000 & 0.349 \\
8.45 & 0.0788 & 0.1725 & 0.0522 & 0.3036 & 0.000 & 0.000 & 0.354 \\
\hline
\end{tabular}

are obtained for other quantities, such as the average magnetization, which are reported in Tables 1 and 2 . We also investigated the twisted flower state, which in agreement with HK we found to have a lower energy than the symmetric flower in this range of $\lambda$ 's. Our result for the transition between twisted flower and vortex, obtained with the same method, is $\lambda_{T F-V}=8.503 \pm 0.005$, whereas HK had found the same transition at $\lambda_{T F-V}=8.57$. The magnetization and energy data for the twisted vortex are given in Table 3.

In comparing with previous results, a note on the different methods used is needed. Our results are based on finite elements on an unstructured tetrahedral mesh of the interior and an explicit resolution of Laplace's equation in the outside. RFH instead used a regular grid in the cube with finite differences, and FFT to evaluate the magnetostatic contributions. Although the method is completely different, we reproduce their results with very good precision. The approach of HK on the other hand, was similar to ours for the interior mesh, but had a different treatment of the outer-space problem, based on a nonlinear mapping to a finite region, and a variational formulation based on optimizing over all solenoidal fields. This can possibly explain the small but noticeable difference in evaluating the magnetostatic energies (our values are systematically lower by about $5 \times 10^{-3}$ than 
TABLE 3. Twisted vortex state. Same data as Table 1. The point at 8.42 has larger numerical errors (by a factor of $3 \div 5$ ) than the others.

\begin{tabular}{c|lllllll}
\hline$\lambda$ & $\varepsilon_{\mathrm{d}}$ & $\varepsilon_{\mathrm{x}}$ & $\varepsilon_{\mathrm{k}}$ & $\varepsilon_{\text {tot }}$ & $\left\langle m_{x}\right\rangle$ & $\left\langle m_{y}\right\rangle$ & $\left\langle m_{z}\right\rangle$ \\
\hline 8.528 & 0.2354 & 0.0443 & 0.0213 & 0.3011 & 0.000 & 0.89 & 0.000 \\
8.489 & 0.2401 & 0.0418 & 0.0197 & 0.3016 & 0.000 & 0.90 & 0.000 \\
8.42 & 0.2484 & 0.0370 & 0.0167 & 0.3022 & 0.000 & 0.91 & 0.000 \\
\hline
\end{tabular}

those reported by HK), which seems to be the main cause of the slight differences in the localization of the critical values of $\lambda$.

In summary, our results lie well within the range of already published values, being in particularly good agreement with those of Rave, Fabian and Hubert [21].

\section{SWitching IN SMALL PARTICLES}

As a second application, we considered switching of small particles. The celebrated Stoner-Wohlfarth (SW) model assumes the magnetization to be uniform inside the magnetic sample, and is valid in the limit of strong exchange, or equivalently small particles. The magnetostatic energy is then a quadratic form in the magnetization, which is a unit vector. For a cube, it is a constant (by symmetry), for more general shapes it is the magnetostatic energy of a suitable "reference ellipsoid". We investigate the geometric effects not captured by the SW approximation, in that we analyze switching in small cubical particles along different directions. We assume a small uniaxial anisotropy along the $z$ axis. The geometry is illustrated in Figure 3. The SW result for both the cube and the smoothed cube is:

$$
h_{\text {crit }}(\theta)=2 K_{u} \sqrt{\sin ^{6}(\phi)+\cos ^{6}(\phi)}
$$

where $\phi$ gives the direction of the equilibrium magnetization and is determined by $\tan ^{3} \phi=\tan \theta$, and $K_{u}=0.05$ is the anisotropy factor. Angles are measured with respect to the easy axis, which we take to be along one of the axes of the cube.

For each direction, we initialize the magnetization in the flower state (i.e. almost uniform magnetization along the easy axis) with orientation opposite to the projection of the applied field on the easy axis, and slowly increase the field until an instability is observed numerically. States are labeled as stable if the residuum decreases below the threshold we established for convergence, and unstable if at some point along the minimization the projection of the average magnetization on the easy axis changes sign (this avoids the unnecessary numerics which would optimize the flipped state). To accelerate the convergence, not all fields are tested, but a bisection method is used around the region where the critical value is expected. Every time, the minimization is started from the most recent stable state with the same orientation, which simulates the progressive increase of the applied field. Errors in this procedure arise both from grid-size effects and from the finite threshold we imposed for convergence. We checked both, by doubling the number of points and dividing the threshold by 10, and results did not change significantly for $\lambda$ up to 6 . For larger $\lambda$ instead a much larger fluctuation has been observed, even if the trend remains stable.

Figure 4 shows the critical field for different orientations. The difference between the crosses and the circles indicates the dependence of the critical field on the azimuth, which cannot be recovered by modeling the cubic particle with an ellipsoid. This difference disappears for fields close to the easy $(y)$ axis, since both planes intersect the easy axis. For both the $\lambda=4$ and the $\lambda=6$ cube, the critical fields at small elevation are significantly larger than predicted by SW. On the other hand, different behavior between the two planes is observed for fields close to orthogonal to the easy axis (i.e. almost contained in the $x$ - $z$ plane).

Figure 5 shows the dependence of the critical field for a given angle (azimuth 0 , elevation $3 \pi / 32$ ) on the cube size $\lambda$. For small $\lambda$ our results reproduce the SW result. With increasing cube size the critical field 

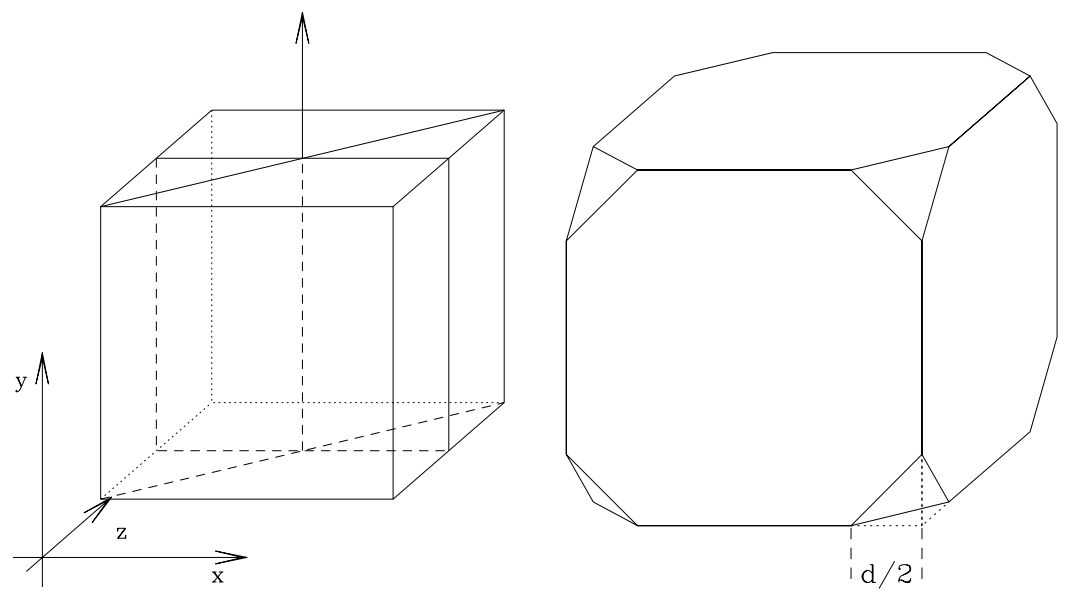

Figure 3. Geometry. The left panel shows the standard cube. The arrow parallel to the $z$ axis indicates the easy axis. The two cross-sections of the cube indicate the planes along which the critical field is plotted in the following figures. The right panel represents the cube with smoothed edges. $d$ is the fraction of the original side length which is removed (i.e. one tetrahedron with edge $d / 2$ is removed from each vertex). For $d=1$ we get a regular octahedron.
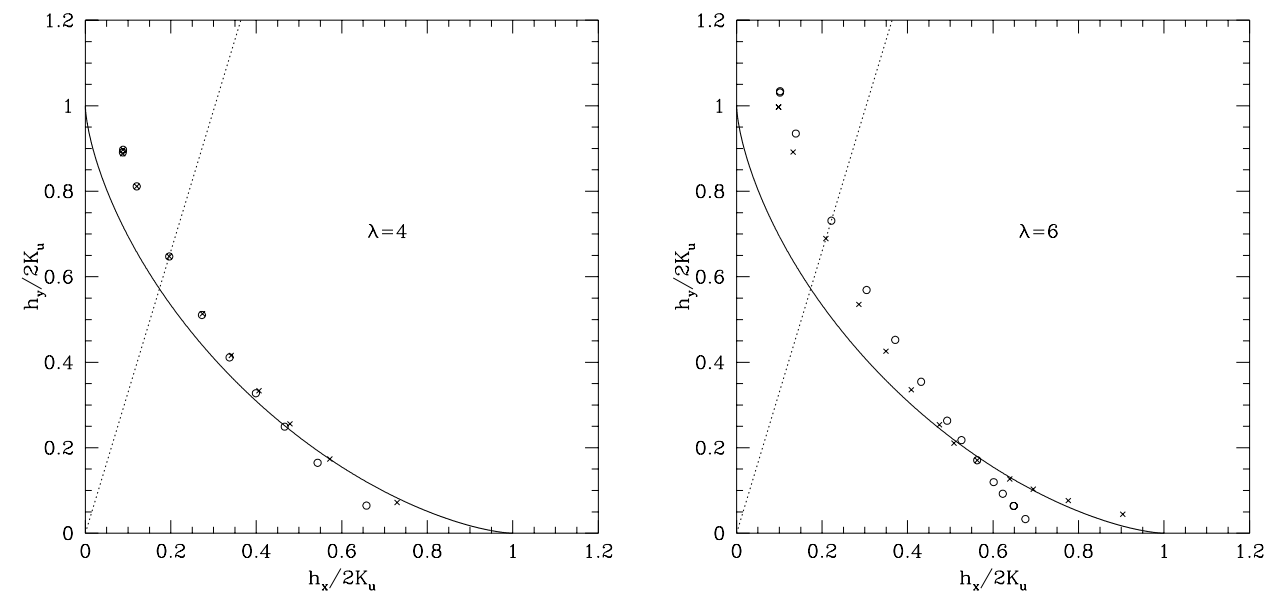

Figure 4. Critical fields for cubes with $\lambda=4$ (left panel) and 6 (right panel), compared with the SW model (full curve). The easy axis is vertical. Circles correspond to points computed in a plane parallel to two faces, crosses to points computed in a plane parallel to two face diagonals (see Fig. 3 for the geometry). The other quadrants are symmetric. The dotted lines indicate the direction selected for the plot in Figure 5.

becomes larger, reaches a maximum for cube sizes around 7, then drops rapidly. The drop is associated with the appearance of complex switching patterns. Indeed, for smaller $\lambda$ the total magnetization remains essentially constant to a value close to 1 during the switch, whereas for larger $\lambda$ it is strongly reduced in the intermediate stages of the transition. We observe that the flower-vortex transition without external field with the value of $K_{u}$ used here takes place for $\lambda \sim 8.2$, significantly larger than the cube size where the critical fields drop. The non-monotonic behavior of the critical field agrees qualitatively with the results of Shabes and Bertram [23]. 


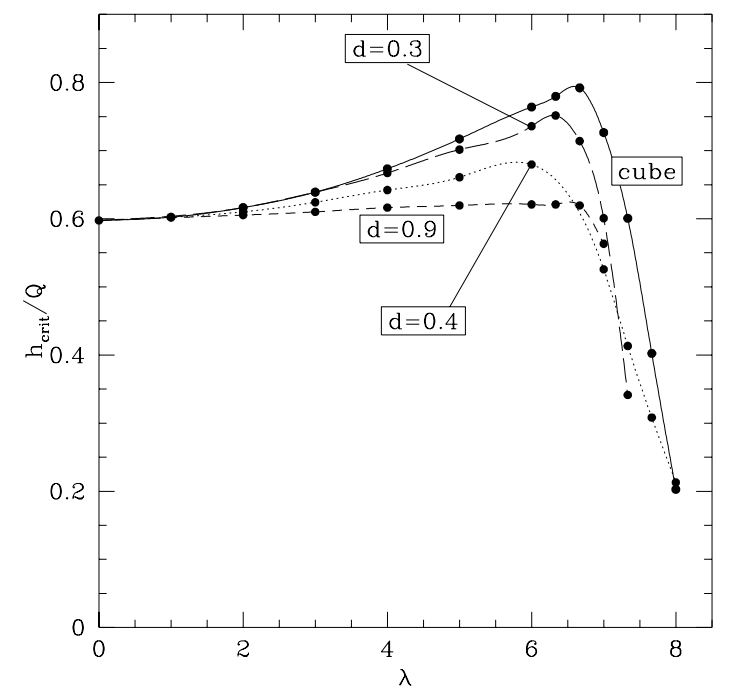

Figure 5. Critical field in direction $\theta=13 \pi / 32$ as a function of $\lambda$. The point at $\lambda=0$ marks the SW result, $h_{\text {crit }} / 2 K_{u}=0.59$. The other points are the computational results, the lines are just a guide for the eye. The highest curve (at intermediate $\lambda$ ) corresponds to a cube, the other to modified cubes with $d=0.3,0.5$ and 0.9 . Up to $\lambda=6$ the estimated discretization error is of the order of the symbol size, for larger $\lambda$ is grows up to 0.1 (in the scale of the figure).

The ellipsoidal approximation on the other hand yields different results: the stability field is constant up to a certain value, then decreases as $1 / \lambda^{2}$.

To better elucidate the role of sample geometry in these computations, we plot in the same figure the results obtained with a cube with smoothed edges, as illustrated in Figure 3, with three different degrees of smoothing. The results for $d=0.3$ are very similar to those of a cube. Instead for $d=0.9$, which is the maximum smoothing we included, we get an almost unnoticeable increase of $h_{\text {crit }}$ with $\lambda$, and hence much closer agreement with the ellipsoidal approximation.

In summary, we studied numerically the influence of non-ellipsoidal geometry on switching fields in small particles. We obtain a significant dependence on azimuth for particle sizes below the multidomain transition, and a non-monotonic dependence on particle size of the switching field for a given orientation.

Acknowledgements. This work was partially supported by the EU TMR network Phase Transitions in Crystalline Solids, contract FMRX-CT98-0229 and by the Deutsche Forschungsgemeinschaft through the Schwerpunktprogramm 1095 Analysis, Modeling and Simulation of Multiscale Problems. Use of the computational resources of the Max Planck Institute for Mathematics in the Sciences, Leipzig, is gratefully acknowledged. Moreover, the three dimensional meshes used in this work have been generated with the software $g h s 3 d$ developed by the INRIA (France). We thank in particular Pascal Frey for having provided us with this software without charge.

\section{REFERENCES}

[1] A. Aharoni, Introduction to the theory of ferromagnetism. Oxford Ed., Clarendon Press (1996).

[2] A. Aharoni, Angular dependence of nucleation by curling in a prolate spheroid. J. Appl. Phys. 82 (1997) 1281-1287.

[3] F. Alouges, A new algorithm for computing liquid crystal stable configurations: the harmonic mapping case. SIAM J. Numer. Anal. 34 (1997) 1708-1726.

[4] F. Alouges, Computation of demagnetizing field in micromagnetics with the infinite elements method. ESAIM: COCV 6 (2001) 629-647.

[5] A. Bagnérés-Viallix, P. Baras and J.B. Albertini, 2d and 3d calculations of micromagnetic wall structures using finite elements. IEEE Trans. Magn. 27 (1991) 3819-3822. 
[6] G. Bertotti, Hysteresis in magnetism. Academic Press, San Diego (1998).

[7] E. Bonet, W. Wernsdorfer, B. Barbara, A. Benoît, D. Mailly and A. Thiaville, Three-dimensional magnetization reversal measurements in nanoparticles. Phys. Rev. Lett. 83 (1999) 4188-4191.

[8] W.F. Brown, Criterion for uniform micromagnetization. Phys. Rev. 105 (1957) 1479-1482.

[9] T. Chang, J.-G. Zhu and J.H. Judy, Method for investigating the reversal properties of isolated barium ferrite fine particles utilizing magnetic force microscopy (mfm). J. Appl. Phys. 73 (1993) 6716-6718.

[10] W. Chen, D.R. Fredkin and T.R. Koehler, A new finite element method in micromagnetics. IEEE Trans. Magn. 29 (1993) $2124-2128$.

[11] Y.M. Chen, The weak solutions to the evolution problems of harmonic maps. Math. Z. 201 (1989) 69-74.

[12] A. DeSimone, Hysteresis and imperfection sensitivity in small ferromagnetic particles. Meccanica 30 (1995) 591-603.

[13] D.R. Fredkin and T.R. Koehler, Finite element methods for micromagnetics. IEEE Trans. Magn. 28 (1992) 1239-1244.

[14] E.H. Frei, S. Shtrikman and D. Treves, Critical size and nucleation field of ideal ferromagnetic particles. Phys. Rev. 106 (1957) 446-454.

[15] R. Hertel and H. Kronmüller, Finite element calculations on the single-domain limit of a ferromagnetic cube - a solution to $\mu$ mag standard problem no. 3. J. Magn. Magn. Mat. 238 (2002) 185-199.

[16] A. Hubert and R. Schäfer, Magnetic domains. Springer, Berlin (1998).

[17] Y. Ishii, Magnetization curling in an infinite cylinder with a uniaxial magnetocrystalline anisotropy. J. Appl. Phys. 70 (1991) $3765-3769$.

[18] R.D. McMichael, Standard problem number 3, problem specification and reported solutions, Micromagnetic Modeling Activity Group, www.crcms.nist.gov/ rdm/mumag.html (1998).

[19] A.J. Newell and R.T. Merrill, The curling nucleation mode in a ferromagnetic cube. J. Appl. Phys. 84 (1998) $4394-4402$.

[20] R. O'Barr, M. Lederman, S. Schultz, W. Xu, A. Scherer and R.J. Tonucci, Preparation and quantitative magnetic studies of single-domain nickel cylinders. J. Appl. Phys. 79 (1996) 5303-5305.

[21] W. Rave, K. Fabian and A. Hubert, Magnetic states of small cubic particles with uniaxial anisotropy. J. Magn. Magn. Mat. 190 (1998) 332-348.

[22] F. Rogier, S. Labbé and P.Y. Bertin, Schéma en temps et calcul du champ démagnétisant pour le micromagnétisme. NUMELEC'97, École Centrale de Lyon (1997).

[23] M.E. Schabes and H.N. Bertram, Magnetization processes in ferromagnetic cubes. J. Appl. Phys. 64 (1988) $1347-1357$.

[24] E.C. Stoner and E.P. Wohlfarth, A mechanism of magnetic hysteresis in heterogeneous alloys. Phil. Trans. R. Soc. London Ser. A 240 (1948) 599-642.

[25] A. Thiaville, Coherent rotation of magnetization in three dimensions: a geometrical approach. Phys. Rev. B 61 (2000) 12221.

[26] L.A. Ying, Infinite elements method. Beijing University Press (1995).

To access this journal online:

www.edpsciences.org 